\title{
An Efficient Coverage Greedy Packet Stateless Routing (EC-GPSR) in Authenties Based Wireless Sensor Networks
}

\author{
V. Shanthi' ${ }^{1}$ D. Somasundareswari² \\ ${ }^{1}$ Department of ECE, Park College of Engineering and Technology, Coimbatore, India \\ ${ }^{2}$ Maharaja Institute of Technology, Coimbatore, India \\ Email: shanthi_v2@yahoo.co.in
}

How to cite this paper: Shanthi, V. and Somasundareswari, D. (2016) An Efficient Coverage Greedy Packet Stateless Routing (EC-GPSR) in Authenties Based Wireless Sensor Networks. Circuits and Systems, 7, 4291-4307.

http://dx.doi.org/10.4236/cs.2016.714351

Received: April 15, 2016

Accepted: April 25, 2016

Published: December 20, 2016

Copyright $(9) 2016$ by authors and Scientific Research Publishing Inc. This work is licensed under the Creative Commons Attribution International License (CC BY 4.0).

http://creativecommons.org/licenses/by/4.0/

\begin{abstract}
A WSN (wireless sensor network) consists of lakhs of sensor nodes with low level energy, memory management, and computation routing capability. The real time world applications of WSN in some extreme perceive environment arrange sensor nodes complex to exchange once they use up the resource. Hence, lots of researchers in this field going towards on how to design a property routing protocol with extension route procedures to safety of transmission with prolong the life span of the network. The classical hybrid protocols such as LEACH and GPSR have better performance in saving the power consumption. However, the choosing formula eliminates the change of nodes' Route will make the nodes acting as cluster heads too many times die of route with power early expressions to the consumption of too much Power and saves the route path. In this paper, we present WSN network route extension with supporting of cloud architecture of the state-of-the-art routing techniques in WSNs. Our Proposed Research belongs overcome of GPSR (Greedy Perimeter Stateless Routing) to EC-GPSR (Efficient Coverage-GPSR), ERA (Efficient Route Autonomous) and IC-GPSR (Isolated Coverage-GPSR) with solving of Route problems due to Route extension with help of cloud to store availability of routes and improve the scalability with compare AODV.
\end{abstract}

\section{Keywords}

GPSR, Route Extension, LEACH, ERA, IC-GPSR, Cloud, WSNs

\section{Introduction}

A wireless sensor network (WSN) is a wireless network available of spatially dis- 
tributed autonomous devices using sensors to monitor physical or environmental conditions (National instruments). A WSN Performing incorporates a gateway that provides wireless connectivity back to the wired real time world and disbursement of nodes. The wireless protocol you select depends on your application and advantage requirements. Some of the available standards include 2.4 $\mathrm{GHz}$ (open sources) radios based on either (Wi-Fi) and applicable in Wimax standards or proprietary radios, which are usually $900-1800 \mathrm{MHz}$. GPSR based hybrid protocol have twice mode of Process: Greedy and Perimeter protocols. GPSR, packets are indicated by their actual with their destinations' Positions. As a result, a forwarding node can make a nearly optimal, greedy choice in choosing a packet's next hop [1]. Specifically, if a node knows its radio Friends node locations, the locally optimal choice of next hop is the Friend node geographically closest to the packet's Final end. If Forward and reverse route could be failure means switch over to perimeter choice in this concern route design and safety considerations are need to development of route.

In Proposed system EC-GPSR (Efficient Coverage-GPSR) states that EC-GPSR require each node periodically (for every one second,) broadcast a hello message containing its latest Position information to its friend node to form path [2]. A Hello message broadcast by a node includes the node ID, sequence No., the current $\mathrm{X}$ and $\mathrm{Y}$ coordinate Position of the node (for in GPSR) and the fraction of the coverage route range and power currently available at the node (for ECGPSR alone). EC-GPSR provides better coverage of nodes and along with individual power of every node.

Routing strategies and WSN route models have recently received a great deal of focusing The importance of Route maintance is done to monitor the Various security strategies are available, such as trust [3] and ReTrust, as well as other types of Information mechanisms and answers that also include single-hop communication as Presented in Many of the data-routing protocols used in WSNs are easy to identify of the attacks. In such a WSN, an adversary can deploy is friend node(s) to generate various types of attacks for denial of service (DOS) or to compromise existing nodes. Through these satisfied nodes, the adversary can perform data network-layer attacks, which predominantly involve the calculation of important data. Attacks that attempt to manipulate data can be categorized into two classes: in the primary, the attacker attempts to influence the user data directly, and in the second, the attacker attempts to affect the core data-routing topology.

Efficient Autonomous Routing (ERA) can be defined as well transmitting data over the network, similarly considering the other factors such as internal and external factors, etc. [4]. The four important factors to be considered are energy cost, robustness, throughput and delay.ERA provides safer mechanism to ECGPSR that means some coverage issues and individual node power can be boost up and save the Route paths with nodes. Main importance of ERA based proto- 
cols finds correct paths and very longing network lifetime by being energy-aware or by increasing residual energy uniformly across the network [4].

A WSN is performed in a Segmentation of favour to control certain environmental conditions or changes in an application. [5] sensing model is widely used due to its simplicity and the fact that it enables theoretical abstraction and analysis. In this, IC-GPSR is used to isolated coverage and safety issue of nodes and network and its overcome the issues of ERA and EC-GPSR. Main importance of IC-GPSR provides security issues to route along with Node. More authenties are applied to establish the network then stack to in cloud to performing the network. Figure 1 states that A Wireless Sensor Network topology with energy of Route efficient.

Let $\mathrm{n}$ Physical nodes be uniformly non metropolitan distributed in a rectangular emer eld at $\left(x_{1} ; y_{1}\right) ;\left(x_{2} ; y_{2}\right) ; \ldots ;\left(x_{n} ; y_{n}\right)$ respectively, and base station be deployed at $(x ; y)$ as shown in Figure 1 The Euclidean distance between the base station and the physical nodes are $d_{1} ; d_{2} ; d_{3} ; \cdots ; d_{n}$ respectively. Here $d_{i}$ is

$$
P / d_{i}=\left(x x_{i}\right)^{2}+\left(y y_{i}\right)^{2}
$$

Case $1\left(d_{i}<d_{0} ; 8 i\right)$ : Let $d^{2}$ be the sum of squares of all the Euclidean distances. As all the nodes will have only free space loss, energy consumption in amplication, $E_{d} 2$ in the sensor network will be sum of amplier energy consumption of the all the nodes.

$$
E_{d} 2=L_{f_{f}}:\left(d_{1}^{2}+d_{2}^{2}+d_{3}^{2}+\cdots+d_{n}^{2}\right)
$$

Sum of square of all the Euclidean distances, $d^{2}$ will be minimum at the centroid of nodes [5]. Thus amplication energy consumption $E_{d} 2$ will also be minimum if base station is placed at the centroid.

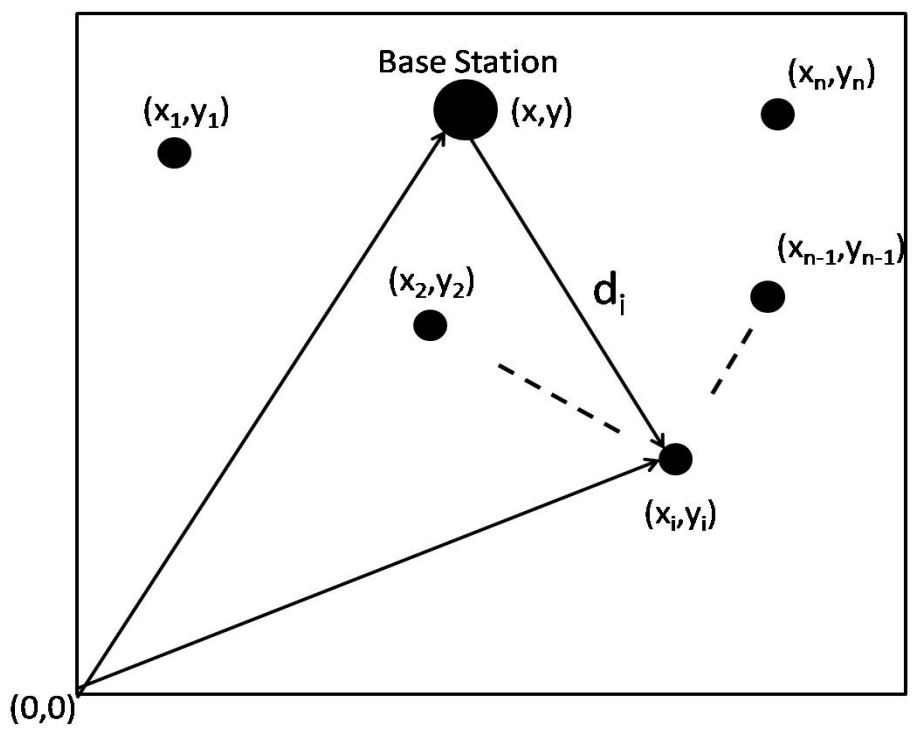

Figure 1. A wireless sensor network topology. 
A number of familiar nodes (sink points) disbursement across the WSN area constitute a cloud. We refer this type node as "cloud node", which is equipped with more resources to save data and acquisition of all data from scalability [6] benefits. Furthermore, a cloud node acts as a sink for sensors nearby due to cloud In order to differentiate from the term "Group of nodes" in classical WSN, These sensors in a zone are organized as independent Near WSNs (NWSN), and all NWSN are merged together by the cloud. The cloud can be viewed as a "virtual sink (vs)" for the whole sensor network. The paper is organized as follows. Section 2 summarizes the existing GPSR and proposes ECGPSR schemes in WSNs. Section 3 describes the working of the proposed ERA. Section 4 provides the information analysis of the scheme IC-GPSR and stack in cloud along with simulation results. Section 5 states the conclusions and future scope of the scheme

\section{Related Work}

The analysis and establishment on the EC-GPSR protocol has been in serious trouble previous decades. Wireless sensing element networking has emerged as a promising technology within the giftera that nodes move and communicate with one another with none centralized management or base stations. Degui Xiao et al. [6] planned associate moving direction and speed into GPSR in creating packet forwarding choices is herewith planned. Key data structures were designed and MOVE was wont to construct typical Grid map urban simulation scenario. The protocol was simulated mistreatment NS-2 below the urban simulation state of affairs and was compared with AODV and GPSR protocols.

P. Samundiswary et al. [1] "Geographical routing protocols of wireless sensing element networks are developed while not considering the security aspects against these attacks. during this paper, a secure routing protocol named secured greedy perimeter homeless routing protocol (S-GPSR) is planned for mobile sensing element networks by incorporating trust based mostly mechanism within the existing greedy perimeter homeless routing protocol (GPSR).Antonio foneseca [10], "Implementation of position-based routing moduleon NS3" routing is seen as associate adequate strategy to mitigate the issues brought by the high quality of the nodes. Greedy Perimeter Stateless Routing is one among the foremostrepresentative protocols of this approach, because the others share a similar basic principle but with enhancements to elements of its methods and GPSR and therefore the conception of location service, that is needed for position-based routing to figure two. A. R. Naseer et al. [2] "name System based mostly TrustEnabled Routing for Wireless sensing element Networks" created analysis on WSN is that the provision of secure routing. Nidhi H. Khunt et al. [3] an Enhance Approach of Route choice Technique of GPSR victimization Multi-Path Mechanism. GPSR uses the position of the supply node and destination node to forward packets. This protocol uses the 2 methods for packet forwarding: Gree- 
dy Forwarding and Perimeter Forwarding. The projected system uses the modify beacon packet strategy that embrace location data and residual energy data. The node will be chosen as forwarding node that is nearest to supply node and that having the lot of residual energy. Min Chen et al. [4] projected One node is chosen as a next hop according to the applied routing strategy, e.g. distance-based strategy or direction-based strategy. This paper proposes a virtual coordinates based mostly routing (VCR) theme, that adopts a new criterion supported virtual coordinates reborn from the absolute coordinates. The results of in depth simulation experiments show that tape recorder achieves AN economical trade-off between energy consumption and end-to-end delivery latency. The overall performance of tape recorder in terms of energy consumption and end to-end delivery latency is healthier than that of each standard distance-based and direction-based algorithms. Kevin C. Lee et al. [5] projected a Geo Cross: A geographic routing protocol within the presence of loops in urban eventualities that Geo Cross exploits the natural plate like feature of urban maps while not resorting to cumbersome planarization. Its feature of dynamic loop detection makes Geo Cross appropriate for extremely WSN. We've got shown that in pathologic cases, Geo Cross's packet delivery quantitative relation (PDR) is systematically above Greedy Perimeter homeless Routing's (GPSR's) and Greedy Perimeter organizer Routing's (GPCR's). We've got additionally shown that caching (Geo Cross + Cache) provides constant high PDR however uses fewer hops. Fabrizio Granelli et al. [6] has projected Associate in Nursing increased GPSR Routing in Multi-Hop transport Communications through Movement Awareness protocol for WSNs that's primarily based represents a modification of well-known GPSR that exploits data concerning movement so as to boost ensuing forwarding node call. Performance analysis of the projected protocol underlines a promising and sturdy basis for planning a routing strategy appropriate for the automotive state of affairs. Dharani N.V. et al. [7] projected a Performance analysis of GPSR Routing Protocol for VANETs mistreatment Bi-directional Coupling-based routing protocol and it's referred to as Greedy Perimeter homeless Routing protocol (GPSR) for VANETs that could be a common position primarily based protocol particularly for routing in MANETs. So as to judge realistic simulation setting bi-directional coupling of OMNET ++/ INET Framework and grappling is chosen for Nagarbhavi region in Bengaluru, India. The simulations area unit finished varied eventualities realizing the impact of quality parameters on routing mistreatment GPSR, and performance is measured in terms of packet delivery quantitative relation and turnout.

Ying Zhang et al. [8] proposed a "Message-initiated Constraint-Based Routing for Wireless Ad-hoc detector Networks". This thesis describes general message specification mechanism to expressly encipher the routing destinations, constraints and objectives in messages, so all-purpose rather than objective or destination specific routing methods will be applied. Mistreatment all-purpose 
routing methods whereas specifying quality-of-service (QoS) properties at the appliance layer expressly in messages, QoS-aware methods for individual messages area unit obtained. We tend to conjointly propose 2 frameworks of allpurpose routing methods for this sort of message specification.

Handkerchief Bhatia, Neha Sood, et al. [9] Hope projected "a replacement Routing Protocol for MANET" This thesis projected a special kind of protocol utilizes scale back network overhead, power consumption, Multi-user Interference (MUI), and supply link responsible ness in changed AODV. within the response of this protocol solely nodes set within the space between the supply \{and the and therefore the/and conjointly the\} destination answer a RREQ packet so as to limit flooding of RREQ packets and thus scale back the overhead and also packet meddlesome one amongst the most drawbacks of the protocol is overhead, Routing Strategy and Routing Maintenance and could be a major problem in several position primarily based routing applications.

\section{Efficient Coverage Greedy Perimeter Stateless Routing Protocol (EC-GPSR) Scheme}

The most well understood tricks to assist with quantifiability area unit hierarchy and caching. Hierarchy doesn't work too well in dynamic ad-hoc wireless networks as a result of it's supported well-defined and rarely-changing boundaries. Caching is beneficial (seen in DSR) however it's conjointly not as scalable as exploitation solely native data (though GPSR will get pleasure from piggybacking). Here, the most idea: use location data to enhance quantifiability. Aim for quantifiability, for each factor on top of. Use the subsequent measures for the quantifiability of a system: Routing protocol message value, Application packet delivery success rate, Per-node state Geographic routing permits routers to be nearly "stateless". Needs propagation of topology information \{for solely for less than for under\} one hop: every node must keep track of only its immediate neighbors' positions. "Self-describing nature" of position is that the key to geography's utility in routing: the position of a packets' destination and positions of the candidate next hops area unit enough to create correct forwarding selections. ECGPSR belongs to Forward and reverse route analysis and maintains of property avoid broken links this projected protocol states that that node is accomplished and node removed neighborhood node takes the responsibility communicate with sink node. This data approved by supply management. Forward and reverse principles area unit maintain the routing data and track the trail. during this manner protocol identifies the initialisation configuration and observance of routing path.

\subsection{Route Design}

Assume that there square measure $\mathrm{n}$ nodes in a very wireless network, and therefore the location of every node is on the market [7]. Then we are able to get 
the table of distances between nodes to make a route by exploitation the subsequent formula (1) A node is randomly chosen because the root node. Once initialized, it'll send messages to encircled nodes requesting their IDs and site data. (2) In response to Step (1), the remaining $n-1$ nodes send their IDs and site data to the foundation node. (3) The space table is formed once the foundation node has received all the data of remaining $\mathrm{n}-1$ nodes. Once the table of distances is formed, we have a tendency to use AN improved Dijkstra formula to deduce the optimum path.

\subsection{GPSR Formula}

Step 1: Initialize Trust Level Count.

Step 2: Update the OT in step with the typical packet Forward Delay.

Step 3: Observe the transmission by neigbour nodes.

Step 4: await forwarding of same packet by different node with time.

Step 5: If Packet is forwarded among OT and therefore the contents don't seem to be altered.

Step 6: Generate Positive Trust path alternatively negative trust path.

Step 7: Detection of Route.

Step 8: Connect of Route.

\subsection{Protocol Implementation}

Support for MAC-layer failure feedback can corrected reducing rate of causation native beacons by piggybacking thus route maintained and extension as doable use promiscuous mode of receiving and transmittal broadcast packets

\subsection{Radio Model}

We have assumed constant radio model that has been utilized in earlier works. For the radio hardware, the transmitter dissipates energy to run the transmitter radio physical science and power amplier, and therefore the receiver dissipates energy and route analysis to run the receive radio the scanerios of the free area ( $\mathrm{d} 2$ power loss) and therefore the multi path attenuation (d4 power loss) channel models were used reckoning on the gap between the transmitter and therefore the receiver [8], If the gap is a smaller amount than a threshold, the free area (fs) model is used; otherwise, the multi path $(\mathrm{mp})$ model is employed.

In the radio model of American state amplier, we have a tendency to use $=\mathrm{a}$ pair of for complimentary area and = four for multipath model. so if a node transmits L variety of bits, the energy utilized in transmission are..

$$
\begin{gathered}
E_{T X}(L ; d)=E_{\text {elec }}: L+E_{\text {amp }}(L ; d) ; \\
L: E_{\text {elec }}+L: f s: d^{2} \text { if } d<d_{0} \\
E_{T X}(L ; d)=n \quad L: E_{\text {elec }}+L: \mathrm{mp}: d^{4} \text { if } d>d_{0}
\end{gathered}
$$


Here threshold

$$
d_{0}=r
$$

To receive $L$ message bits, the radio spends energy

$$
E_{R X}(L)=E_{\text {elec }}: L:
$$

The above radio model involved in the EC-GPSR protocol.

\subsection{Deployment Strategy}

The readying strategy relies chiefly on the kind of sensors and also the application. The subsequent deployments methods area unit usually employed in WSNs. Random readying: Random deployment is that the most sensible approach of inserting the sensing element nodes. For a dynamic sensing element network, [9] where there's no a-prior data of best placement, random readying could be a natural possibility. Incremental deployment: The progressive placement strategy could be a centralized, one-at-a-time approach to position the sensors. The implementation makes use of knowledge gathered through the antecedently deployed nodes to work out the perfect readying location of successive sensing element node.

\subsection{Routing of EC-GPSR}

Routing within the WSNs is difficult attributable to the inherent characteristics that distinguish these networks from the opposite wireless networks like unintentional networks or cellular net-works. It's unimaginable to use a worldwide addressing theme for the preparation of a comparatively sizable amount of sensing element nodes. Thus, ancient IP-based protocols might not be applicable to WSNs. [9] sensing element nodes square measure tightly affected in terms of energy, processing, and storage capacities. In most of the appliance eventualities, nodes in WSNs square measure typically stationary once preparation apart from some mobile nodes. Nodes in different ancient wireless networks square measure absolve to move, which ends up in unpredictable and frequent topological changes.

\subsection{Proposed EC-GPSR Algorithm}

Let $\mathrm{n}$ sensing element nodes area unit arbitrarily distributed during a topology.

Step one: each node broadcast a howdy message with its id exploitation TDMA or SDMA planning. Every node calculates $(n-1)$ distances $\left(d_{i j}\right)$ exploitation RSSI from all alternative nodes. Any each node calculates average of all the gap estimates within the routes.

Step a pair of: each node can broadcast and multicasting data and therefore the node having highest Distance average is that the farthest node from the center of mass and can lie at the corner of topology This node are going to be rst reference node for the system. 
Step three: Farthest node from the rst reference node are going to be second reference node This node can invariably belong opposite corner of node one.

Step four: currently node a pair of can send knowledge of all distances $d_{2 j}$ to node one. Node one nds $\left(d_{1 j}+d_{2 j}\right)$ eight $j ; j_{6}=1$; a pair of. The node that has highest worth of this add are going to be the node farthest from one \&amp; a pair of nodes. This node also will belong the corner of topology, shown as node three. This node is going to be third reference node for the system.

Step five: currently node three nd the farthest node from it and this may be fourth reference node. This node can belong opposite corner of node three.

Step 6: Node having minimum Dave can have minimum distance from the center of mass. This node is going to be the fth reference node for our protocol. This node can lie at the middle of topology and act as origin for Route localization or extension.

\subsection{Performance Analysis}

To test the proposed technique, the specifications of the Dynamic and far routes were used for the Nodes. Simulations were conducted with the 5 and 50 nodes selected using the proposed technique. Simulations were undertaken using NS2.3 version from the results it is observed that the Packet delivery ratio decrease in AODV compared to EI-GPSR is $6.75 \%$. LEACH is compared to EI-GPSR is 7.15\%. Then Throughput decrease in AODV compared to EI-GPSR is $3.89 \%$. LEACH is compared to EI-GPSR is $5.27 \%$. The proposed algorithm technique [7] decreased the Packet drop by $1.16 \%$ compared to the AODV and $4.16 \%$ Compared to LEACH. Obtained original and route extension. Similarly, when the simulation was carried End To End Delay decreased by $1.99 \%$ then AODV and $3.25 \%$ then LEACH. Compared to Route extension is increased by $4.08 \%$ then AODV and $1.17 \%$ then LEACH. Further work to improve EC-GPSR through Efficient Autonomous Route (ERA).

\section{Efficient Route Autonomous}

We projected a way to contemplate a stable and reliable economical routing in WSNs. projected methodology uses 2 vital factors together with stability of nodes and dependability operate of nodes to pick next node in routing formation. This methodology selects nodes with most routing path with power and most dependability to extend network life [9]. The projected methodology is flat and reactive routing, as a result of next node selects exploitation neighborhood info and there's no mounted and predefined structure. This methodology may be a distributed so every node equip to property link. We have a tendency to assume all nodes square measure mounted. This algorithmic program includes of 3 phases:

1) Route Identity section: during this section, every node identifies its neighbors in conjunction with stability. Every node sends identity range (ID) and its node id to multi-hop neighbors. With the passage of your time, energy of nodes 
decreases. So, we have a tendency to should repeat this section (e.g. ten seconds). Here, every node informs of ID and energy of route path and range of its neighbors. Then, every node computes the common of its neighbor energy with route path and is termed ERavg. If energy of nodes square measure but ER (Energy Route) avg, it'll not increase the list of neighbor nodes. Within the next section, we have a tendency to reward and punish via ERavg. This section is recurrent just the once in per choice step.

2) Route Configuration section: during this section, in line with dynamic changes of network and nodes Route node state of affairs, $\mathrm{RC}$ has been used with variable structure. A network of the RC (Route Configuration) similarity to the UDG was used. It's shaped through militarization every node to a RC. At every stage of this approach, the RC haphazardly chooses one amongst its actions so an answer will be found within the routing downside. At first, action likelihood is equal $P i=1 / n . R C N$ is neighbors range of every node $\mathrm{I}$ is action range. At first, a node is chosen haphazardly. Then, the neighbors of this node square measure elect as next node. At every stage of this approach, we have a tendency to update nodes set in route. Future node is chosen supported action likelihood. This section continues till a route between supply and destination nodes is found. To pick nodes in route If we've $E_{\mathrm{i}} \geq E_{\text {avg }}$, elect action must [10] be rewarded to worth of a. If we've $E_{i} \&$ lt; Eavg \& lt; RC elect action should be penalised to worth of $b$. At last, once add of nodes in and out route was up to all nodes, current step are going to be finished. For causation reliable information, the projected methodology uses multiple ways and links with high dependability. We have a tendency to show dependability as likelihood of success transition between 2 nodes that $\mathrm{k}$ is sets of routes between supply and destination nodes. The used model relies on In formula one, $P(k, t)$ is dependability of the kth route and Stability can encounter and frame path. Dependability fof every|of every\} route be calculated with multiple of active links in each route

3) Dominant introduce this section, achieved route continues its operation. We have a tendency to use the definition of life in in line with it, life of network is that the time that the route is disconnected. Finally, the simplest route may be a route with most of residual energy and confidence that's outlined as Rreq. Figure 2 states that ERA.

This scheme have little bit locked in safety and security issues so switch over to IC-GPSR.

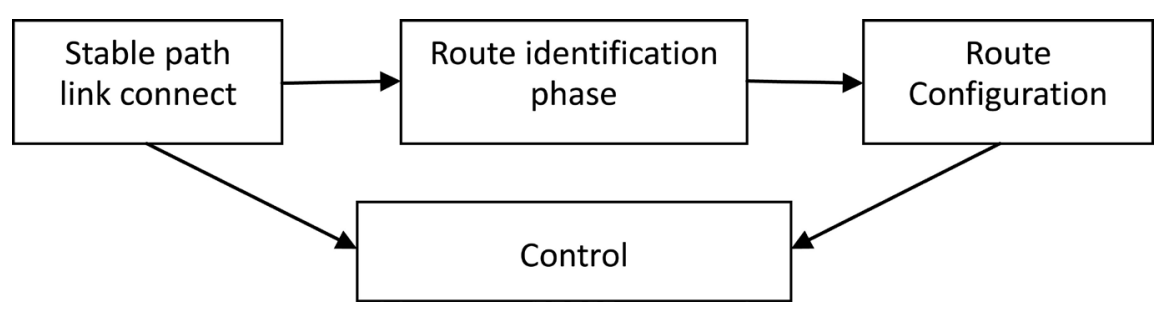

Figure 2. ERA scheme. 


\section{Routing Establishment and Isolated Coverage Based GPSR in Wireless Sensor Networks (IC-GPSR)}

The planned routing theme is predicated on the actual fact that the protection of route mechanism consumed to send a message to a far off node is larger than the energy beside safety required for a brief vary transmission. IC GPSR protocol is extended mistreatment aggregation node or head set node. Isolated Aggregation (IA) node is chargeable for transmission messages to the distant base station and routing is set mistreatment the individual head set members [11]. The head set is set on a routine basis with relevancy the monitor power level of the signal received to the bottom station at the time of reception of "hello packets". At just one occasion, just one member of the top set is active and also the remaining head set members are in sleep mode. The task of transmission to the bottom station is uniformly distributed among all the top set members kind of like AODV and LEACH protocol. Every cluster includes a head set that consists of many virtual cluster heads. The head set is set on a routine basis with relevancy the energy of the signal received to the bottom station at the time of reception of "hello packets". At just one occasion, just one member of the top set is active and also the remaining head set members are in sleep mode.

\subsection{Give Priority to Source Node of Special (IC Algorithm) Packets with Extension}

Step 1: Fill within the essential extension RREQ fields.

Step 2: Broad price of RREQ Message and Maintance of Error.

Step 3: Active route time is about to a continuing and calculate the first route to feature.

Step 3: Reception of RREQ.

Step 4: Check If RREQ is special style of packet then expire already store entry in routing table and store special packet on priority basis, settle for RREQ.

Step 4: Update the routing entry and route discovery otherwise don't trouble.

Forward it to next node.

Step 5: If I'm the destination Send RREP message to the updated reverse rout within the table.

Step 7: Set the Route expire time to current time + active route timeout +original route-Error.

Step 8: Priority basis.

The on top of planned formula states that isolated coverage to produce nodes for economical transmission. Route request offer to network so acquire the Route ACK then extension of RREQ Fields.

\subsection{Cloud}

Stack in cloud development then nodes belong to broadcasting info of safety coverage with Active time route set. The security coverage of route of the pro- 
jected theme is illustrated in one. Authentication problems two. Illegal activity by service providers 3. Attacks on physical security four. Insider abuse of access five. Phishing 6. Compromising the device accessing the detector network. 6. Access management issues 7 . Session hijacking and session riding eight. Network/packet sniffing.

\section{Compare with LEACH and AODV}

Above proposed schemes are comparing with LEACH and AODV with respect Scalability benefits:

LEACH (LOW ENERGY adaptational clump HIERARCHY): As we tend to all grasp that everyone the networks have a precise life throughout that nodes have low energy by exploitation that, the nodes gather, process, and transfer data. This implies that every one aspects of the node, from the sensing element module to the hardware and protocols, should be designed to be very energy-efficient. Decreasing energy usage by an element of 2 will double system life, leading to an outsized increase within the consolidated quality of the system. Additionally, to scale back energy dissipation, protocols ought to be sturdy to node failures, fault-tolerant and ascendable so as to maximise system life. LEACH is that the initial network protocol that uses graded routing for wireless sensing element networks to extend the life time of network. All the nodes in an exceedingly network organize themselves into native clusters, with one node acting because the clusterhead [12]. All non-cluster-head nodes transmit their knowledge to the cluster-head, whereas the cluster-head node receive knowledge from all the cluster members, perform signal process functions on the information (e.g., knowledge aggregation), and transmit knowledge to the remote base station. Therefore, being a cluster-head node is way additional energy-intensive than being a noncluster-head node. Thus, once a cluster-head node dies all the nodes that belong to the cluster lose communication ability. LEACH incorporates irregular rotation of the high-energy cluster-head position such it rotates among the sensing elements so as to avoid exhausting the battery of anybody sensor within the network. During this method, the energy load related to being a cluster-head is equally distributed among the nodes. Since the cluster-head node is aware of all the cluster members, it will produce a TDMA schedule that tells every node precisely once to transmit its knowledge. Additionally, employing a TDMA schedule for knowledge transfer prevents intra-cluster collisions. In this read of LEACH might be little satisfies in EC-GPSR, ERA and IC-GPSR.

AODV (Adhoc On demand distance vector), as a reactive routing protocol AODV uses ancient routing tables, one entry per destination and sequence numbers area unit wont to verify whether or not routing data is up-to-date and to stop routing loops. It helps in each multicasting and unicasting. AODV makes use of try to seek out the route. The supply node broadcast the RREQ i.e. Route Request message to its neighbors to seek out the route to destination. The RREQ 
message contains the supply and destination address, lifetime of message, sequence numbers of supply and destination and request ID as distinctive identification [9]. Destination Sequence range is that the latest sequence range received within the past by the supply for any route towards the destination and supply Sequence range is that the current sequence range to be utilized in the route entry inform towards the supply of the route request. If any node from a listing of neighbors is destination or is aware of the route to destination, it will send RREP message to supply.

\section{Simulation Experiment}

To study the performance of the projected rule, we've conducted many simulation experiments (Experiments 1-5) in 3 projected protocol teams. the primary cluster of experiments evaluates the results of the projected rule supported measurability and also the second cluster of experiments worries with investigation the impact of the route extension of rule on the coverage size and route performance. GPSR protocol was projected to ensure packet causing with dependability. This protocol sends many copies of packet to a number of neighbor nodes. The amount of needed routes is calculated supported channel error and topological data of network. European Economic Community GPSR projected a distributed rule for constructing a backbone in WSN and known as it economical coverage of network. It chooses solely nodes with Route path levels because the member of the virtual backbone. Solely nodes with power levels higher than a predefined threshold may be enclosed within the EC-GPSR ERA projected in Virtual Backbone for Stability for Route economical construction in WSN. ViBES construction enclosed 2 vital phases: 1 ) choice of primary stability nodes 2) their infrastructure to make a connected backbone. Then IC-GPSR it have isolated coverage of links of Path and maintain secure. We have a tendency to compare our projected rule with AODV and LEACH with measurability edges. We have a tendency to assume that the simulation network being employed is in a very place wherever varied events in a very WSN. And following table is Simulation setup for our projected work. Table 1 shows simulation parameters. The simulation parameters used for the simulations area unit given in Table one. The simulation is dispensed for length fifty|of fifty\} seconds and results obtained area unit averaged over 50 runs by victimization totally different seeds.

The arrivals and departures are simulated as independent procedures at regular intervals. The time interval between the messages is set to 0.14 seconds. The following scalability benefits metrics are used to evaluate the schemes.

Througput: The rate of successful message delivery over a communication channel. The data these messages belong to may be delivered over a physical or logical link, or it can pass through a certain network node.

Avarage delay: The time taken by the scheme to configure a node interface with an allocated IP address. 
Table 1. Simulation parameters.

\begin{tabular}{cc}
\hline Parameter & Value \\
\hline Simulation area & $1000 \mathrm{~m} \times 1000 \mathrm{~m}$ \\
Number of nodes & $50-200$ (variable) \\
Mobility Model & Random way point \\
MAC Model & IEEE 802.11 \\
Transmission Range & 250 meters \\
Data Rate & 2 Mbps to 3 Mbps \\
Node mobility & $10 \mathrm{~m} / \mathrm{s}$ \\
Protocol & AODV,GPSR \\
\hline
\end{tabular}

Packet deliver ratio: It is the ratio of the number of successful IP addresses allocated, to the total number of IP address requested at varying population of resource constrained nodes.

Packet loss: The total numbers of control and maintenance packets dropped during the packet exchanges of the scheme.

Figure 3 shows the Throughput Proposed Algorithms of EC, ERA, IC vs AODV \& LEACH when the certain time the total size of useful packets. The average increases in Throughput using EC in routing compared to LEACH is $25.08 \%$ and AODV is by $10.081 \%$. The proposed ERA Increases the overall average Throughput than by $15 \%$ of AODV and $26 \%$ of LEACH. The proposed IC-GPSR is efficiently to compare the Throughput obtained. The obtained throughput by $18 \%$ of AODV and $33 \%$ of LEACH.

Figure 4 shows the Average delay Proposed Algorithms of EC, ERA, IC vs AODV\& LEACH when the certain time the total size of useful packets. The average delay decreases in EC -GPSR in routing compared to LEACH is $28.08 \%$ and AODV is by $12.081 \%$. The proposed ERA decreases the overall average delay than by $14 \%$ of AODV and $28 \%$ of LEACH. The proposed IC-GPSR is efficiently to compare the Throughput obtained. The obtained throughput by $17 \%$ of AODV and $33 \%$ of LEACH.

Figure 5 shows the Packet deliver ratio-Proposed Algorithms of EC, ERA, IC vs AODV \& LEACH when the certain time the total size of useful packets. The average increases in PDR using EC-GPSR routes compared to LEACH is $27.07 \%$ and AODV is by $14.081 \%$. The proposed ERA Increases the overall average Throughput than by $13.75 \%$ of AODV and $27 \%$ of LEACH. The proposed IC-GPSR is efficiently to compare the PDR obtained. The obtained throughput by $17 \%$ of AODV and $33 \%$ of $\mathrm{LEACH}$.

Figure 6 shows the Packet Loss Packet deliver ratio-Proposed Algorithms of EC, ERA, IC vs AODV \& LEACH when the certain time the total size of useful packets. The decreases in packet loss using EC-GPSR routes compared to $\mathrm{LEACH}$ is $25.08 \%$ and AODV is by $12.081 \%$. The proposed ERA decreases the 
overall packet loss than by $14 \%$ of AODV and $26 \%$ of LEACH. The proposed IC-GPSR is efficiently to compare the PACKET LOSS obtained. The obtained

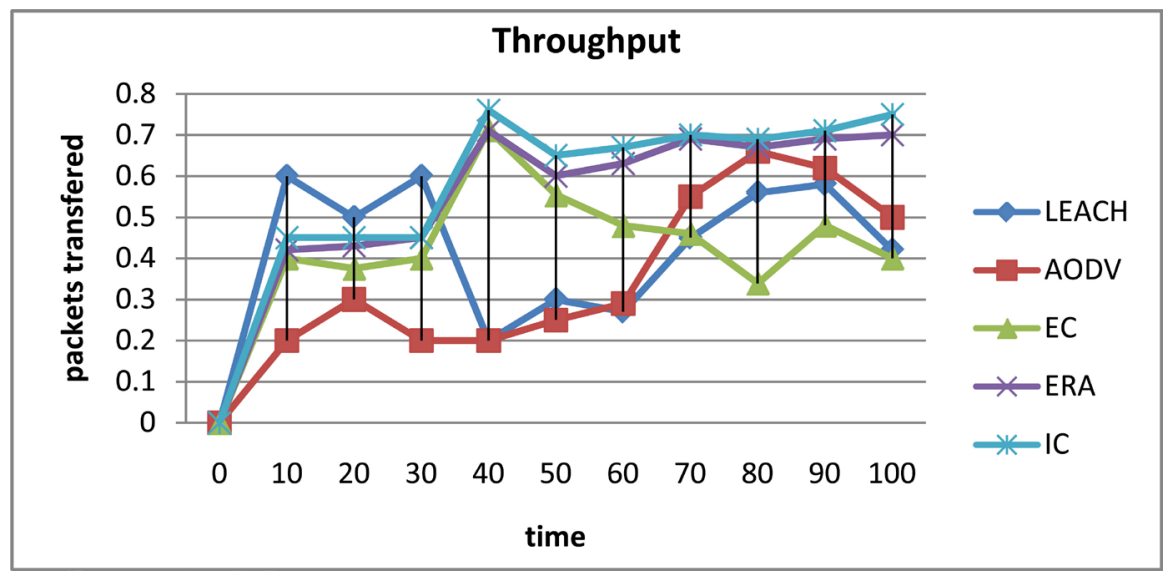

Figure 3. Throughput—Proposed Algorithms of EC, ERA, IC vs AODV \& LEACH.

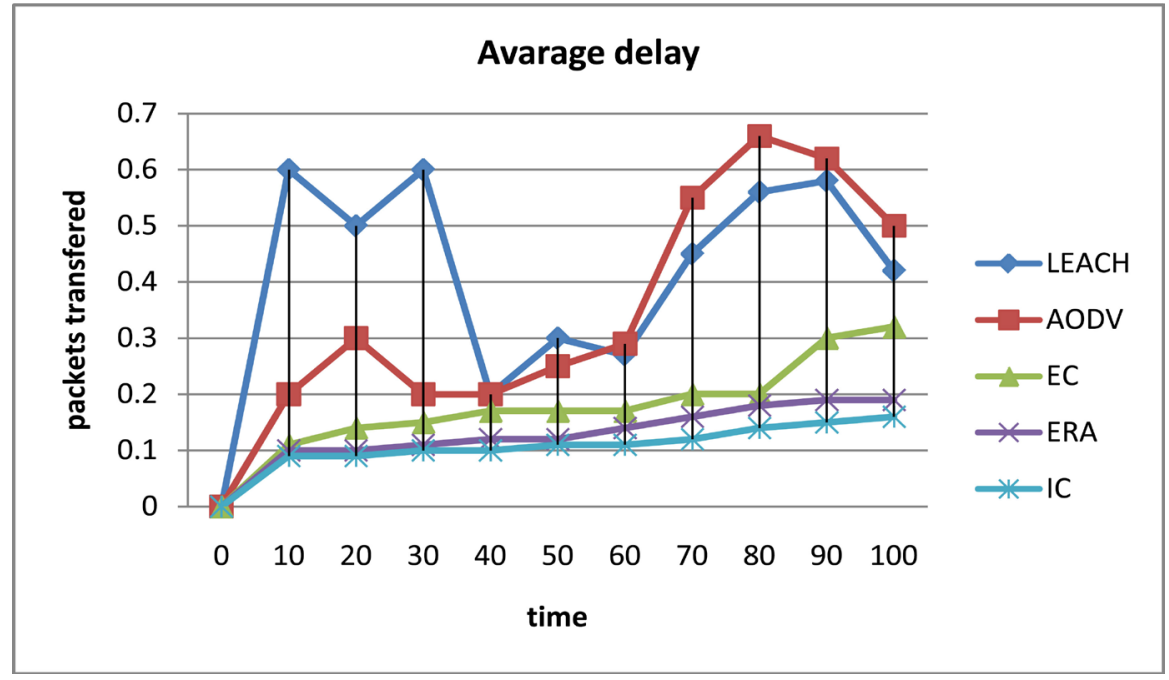

Figure 4. Average delay-Proposed Algorithms of EC, ERA, IC vs AODV \& LEACH.

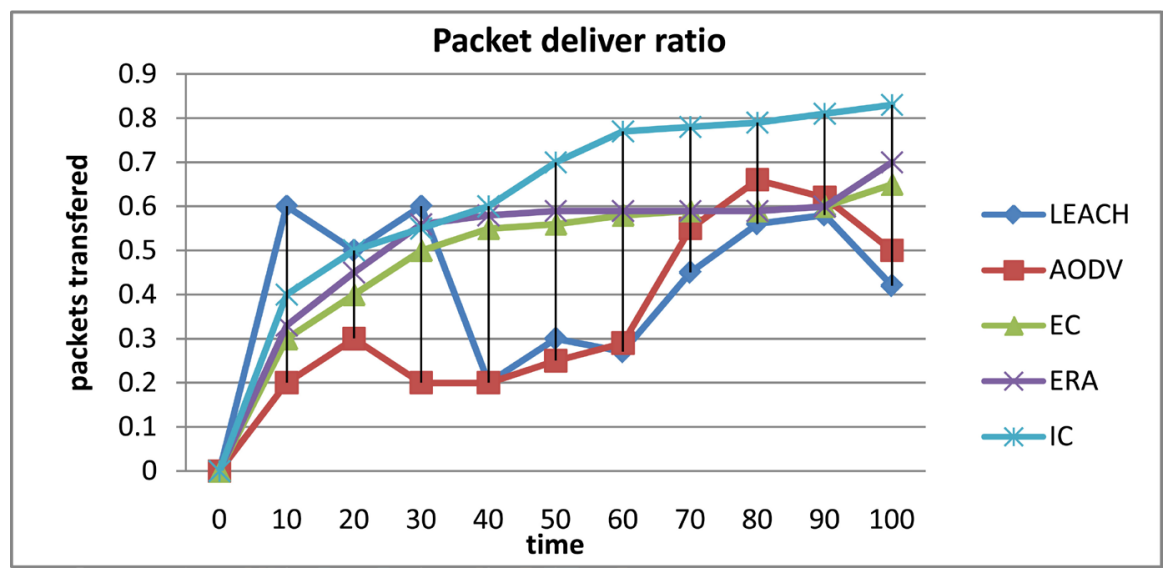

Figure 5. Packet deliver ratio-Proposed Algorithms of EC, ERA, IC vs AODV \& LEACH. 


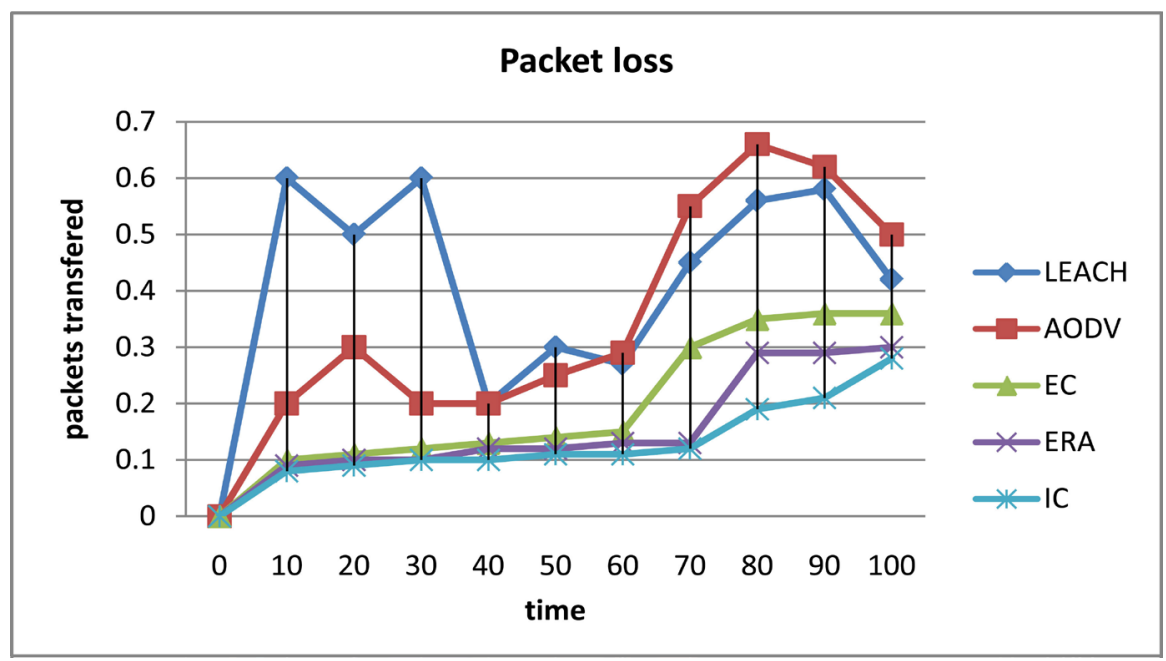

Figure 6. Packet Loss Packet deliver ratio-Proposed Algorithms of EC, ERA, IC vs AODV \& LEACH.

PACKET LOSS by $16 \%$ of AODV and $34 \%$ of LEACH.

\section{Conclusion}

Wireless Sensor Networks make up GPSR based Route extension in Path to compute task mapping to identification of Routes and avoid attacks. In this research, a new concept of efficient integrity and compared with AODV \& LEACH is proposed. It investigated the performance on the basis of packet delivery ratio, throughput, packet drop, and end to end delay its simulated by NS2. It achieved its goal by combining a location-based routing methodology with a link statebased mechanism. Further, it introduced the conception of Autonomous named ERA protocol specially designed for WSN, to deal with the problems of fake path avoidance. The performance analysis shows that, the Packet delivery and throughput was high and expeditiously resolved the Fake paths and safety of node. The projected protocol outperforms the prevailing AODV and LEACH protocol. We have developed a protocol adaptation increased Isolated coverage GPSR Protocol (IC-GPSR) to style a supported a safety measures. The proposed techniques can be investigated for Route extension IOT networks is emerging as a popular mechanism for high rating speed, large data transfer and security. Further investigations can be carried out using the proposed algorithm in Rescue mass Networks.

\section{Acknowledgements}

Thanks to support Department of ECE Park college of Engg and technology Coimbatore to done my Research work.

\section{References}

[1] Samundiswary, P., Sathian, D. and Dananjayan, P. (2010) Secured Greedy Perimeter 
Stateless Routing for Wireless Sensor Networks. International Journal of Ad Hoc, Sensor \& Ubiquitous Computing (IJASUC), 1.

[2] Naseer, A.R. (2013) Reputation System Based Trust-Enabled Routing for Wireless Sensor Networks. IET Wireless Sensor Systems, 1, 74-84.

https://doi.org/10.5772/50736

[3] Khunt, N.H., Kodinariya, T.M. and Sharma, S.S. (2016) An Enhance Approach of Route Selection Technique of GPSR Using Multi-Path Mechanism over MANET. International Journal of Engineering Science and Computing, 6, 4721-4723.

[4] Chen, M., Wang, X.D., Leung, V.C.M. and Yuan, Y. (2006) Virtual Coordinates Based Routing in Wireless Sensor Networks. Sensor Letters, 4, 1-6. https://doi.org/10.1166/sl.2006.039

[5] Lee, K.C., Cheng, P.-C. and Gerla, M. (2010) GeoCross: A Geographic Routing Protocol in the Presence of Loops in Urban Scenarios. Ad Hoc Networks, 8, 474488. https://doi.org/10.1016/j.adhoc.2009.12.005

[6] Granelli, F., Boato, G., Kliazovich, D. and Vernazza, G. (2011) Enhanced GPSR Routing in Multi-Hop Vehicular Communications through Movement Awareness.

[7] Dharani, N.V., Shylaja, B.S. and Ele, S.L. (2015) Performance Evaluation of GPSR Routing Protocol for VANETs Using Bi-Directional Coupling. International Journal of Computer Networks (IJCN), 7.

[8] Zhang, Y. (2009) Message-Initiated Constraint-Based Routing for Wireless Ad-Hoc Sensor Networks.

[9] Bhatia, B. and Sood, N. (2014) AODV Based Congestion Control Protocols: Review. International Journal of Computer Science and Information Technologies, 5, 45704575 .

[10] Asgarnezhad, R. and Nematbakhsh, N. (2015) A Reliable and Energy Efficient Routing Algorithm in Wsn Using Learning Automata. Journal of Theoretical and Applied Information Technology, 82.

[11] Pranitha, P. and Swamy, G. (2011) A Review on Enhanced GPSR Protocol for Wireless Sensor Networks. Computer Engineering and Intelligent Systems, 2. www.iiste.org

[12] Tripathi, R.K. (2012) Base Station Positioning, Nodes' Localization and Clustering Algorithms for Wireless Sensor Networks. 
Submit or recommend next manuscript to SCIRP and we will provide best service for you:

Accepting pre-submission inquiries through Email, Facebook, LinkedIn, Twitter, etc. A wide selection of journals (inclusive of 9 subjects, more than 200 journals)

Providing 24-hour high-quality service

User-friendly online submission system

Fair and swift peer-review system

Efficient typesetting and proofreading procedure

Display of the result of downloads and visits, as well as the number of cited articles Maximum dissemination of your research work

Submit your manuscript at: http://papersubmission.scirp.org/

Or contact cs@scirp.org 